\title{
Entretien
}

\section{Maurice Matieu : inventer un rapport entre peinture, mathématiques et politique?}

\section{Propos recueillis par Nicole Mathieu}

\author{
Maurice Matieu ${ }^{1}$, Nicole Mathieu ${ }^{2}$ \\ 1 Artiste peintre, 8 La Rayrie, 50450 Saint-Denis-le-Gast, France \\ 2 Géographe, UMR LADYSS, Université Paris 1, 2 rue Valette, 75005 Paris, France
}

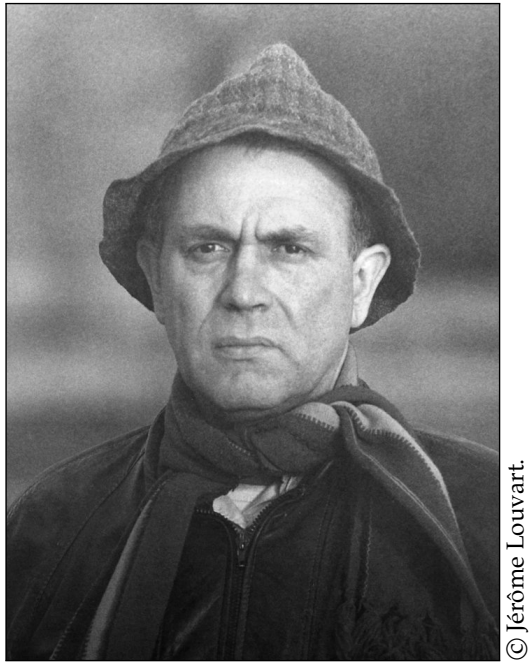

Nicole Mathieu (NSS) : Matieu, vous venez de publier un livre, Posthume sur mesure (2007), qui commence par un entretien avec le philosophe Stéphane Douailler et se poursuit par des textes sur votre travail, écrits par plusieurs intellectuels de renom. En fait, dès cet entretien, on apprend que votre entrée dans la peinture, comme votre éloignement des mathématiques, est liée à votre conception du politique. Mais je voudrais, pour les lecteurs de NSS, essayer avec vous de comprendre ce rapport si particulier que vous établissez entre science et art, entre mathématiques et peinture, et aussi de l'approfondir, au plus loin, du côté du politique. Pouvez-vous déjà me dire ce que vous évoque le terme d'engagement, qu'est-ce que c'est pour vous qu'un intellectuel engagé?

Auteur correspondant : N. Mathieu, mathieu@univ-paris1.fr
Maurice Matieu : L'engagement, je ne sais pas ce que $c^{\prime}$ est et d'ailleurs je ne sais pas ce que je fais. C'est une absence de choix, puisque rien ne le remet en question. Dès le départ, j'ai toujours eu le sentiment d'appartenir au Monde, à la Cité. L'engagement, c'est cette conscience d'appartenance, et rien de plus. Toute la sensibilité que je ressens de cette appartenance, eh bien, je veux en témoigner. Et je veux aussi, par mes actes, avoir la possibilité de le faire savoir. L'acceptation, le refus. Mais je n'accorde aucune valeur particulière au refus.

N.M.: Alors, du coup, j'ai une question qui correspond bien à ce que vous venez de dire. Car, finalement, vous n'avez quand même pas complètement abandonné la dimension sociale du scientifique que vous étiez. Certes, vous avez dit que vous aviez renoncé aux mathématiques pour entrer dans la peinture, mais, dans une certaine mesure, vous n'avez jamais non plus renoncé à cette dimension scientifique. Etj'aimerais que vous me donniez quelques éléments pour comprendre les raisons pour lesquelles vous êtes toujours resté un "scientifique», ne serait-ce que dans l'enseignement des mathématiques, et que vous avez même considéré que vous étiez dans la «Cité » par votre position à l'intérieur de la faculté des sciences de Paris.

Maurice Matieu : Il faut d'abord distinguer le rapport à l'enseignement des mathématiques de celui à l'indiscipline mathématique. J'ai très vite pris conscience que, dès l'instant où je ne maintenais pas une activité de recherche dans le département où j'avais été recruté, je ne pouvais que démissionner. Charles Pisot, mon patron, m'en a dissuadé : «Votre place est ici, on ne sait pas d'où viennent les idées. » Si je voulais garder une place légitime dans l'enseignement supérieur, il fallait que j'invente 


\begin{abstract}
Biographie
Maurice Matieu est né à Paris en 1934. Alors que, dès ses vingt ans, il entame une carrière prometteuse de mathématicien (au Collège de France dans l'équipe de Francis Perrin, puis à la faculté des sciences de Jussieu), la guerre d'Algérie provoque une rupture dans son engagement professionnel. Il s'affirme artiste peintre en 1959 et fait sa première exposition en 1965 : Cinq peintres et un sculpteur à la galerie Maeght de Paris (texte d'Alain Badiou dans la revue Derrière le miroir, éd. Maeght). Depuis cette date, il a fait de très nombreuses expositions personnelles et collectives, en France et à l'étranger, dont on trouve la liste sur ses sites Internet (http://www.laruche-montparnasse.com/mauricematieu/nouveausite/ et http://pagesperso-orange.fr/maurice.matieu/) ainsi que dans son dernier livre, Posthume sur mesure (Éditions du Regard, 2007). Plusieurs écrivains et philosophes ont écrit sur sa peinture (Alain Badiou, Jean Borreil, Barbara Cassin, Stéphane Douailler, Abdelwahab Meddeb, Jacques Rancière, Philippe Sergeant, Juliette Simont, Mathias Tripp, Pierre Verstraeten...). Parmi les expositions collectives qu'il a organisées, citons : Dissonances au Méjan (Arles, 1985) et 7 peintres irakiens à l'Institut du monde arabe (Paris, 2000). Son activité éditoriale est importante. Il a créé en 1989, avec Jean Borreil, « Regard sur le regard » au Collège international de philosophie, rencontre entre philosophes et artistes plasticiens qui constitua par la suite une rubrique de la revue du Collège : Le Cahier du Collège international de philosophie, devenu Rue Descartes, où il publia les «Ateliers » de Jean Ipoustéguy, Eduardo Arroyo, Gilles Aillaud, Anselm Kieffer, Julio Le Parc, Daniel Buren, Guy de Rougemont, Valerio Adami, Takis, Hervé Télémaque. De ses livres, on retiendra aussi La Banalité du massacre (Actes Sud, 2001) et, en collaboration avec Barbara Cassin, Voir Hélène en toutes femmes (Les Empêcheurs de penser en rond, 2000).
\end{abstract}

un enseignement. J'ai pu rester à Paris VI en créant une section, que j'ai appelée la « section travailleurs ». J'en revendique à la fois la paternité et la réussite. Elle a été continuée après mon départ et s'est amplifiée, puisque le département a été témoin d'un succès, à ses yeux, incontournable : un Noir camerounais, gardien de nuit, entré dans cette " section travailleurs » est devenu professeur dans cette université. La possibilité élitiste a pu être reconnue ; la section, au début mal vue, a pu se développer. $C^{\prime}$ est la conscience que l'enseignement ne peut être dissocié d'une recherche qui m'a fait entrevoir une solution sociale. C'est le premier point.

D'autre part, bien sûr, une formation ne s'oublie pas. L'autre point de vue que m'offrait mon activité d'artiste m'a permis de comprendre le sens profond d'une formation scientifique : $c^{\prime}$ est $q u^{\prime}$ on ne réfléchit pas seulement dans son propre domaine. C'est toujours une erreur, je crois, d'avoir une réflexion qui se limite à la direction, unique, dans laquelle on est engagé. Les mathématiques, hier comme maintenant, me servent d'axe de réflexion, d'appui de réflexion, d'enrichissement encore de connaissances, de mouvement d'idée pour une pratique quotidienne qui est maintenant, pour moi, la peinture. Quel$q^{\prime} u^{\prime} u n$ ayant une autre formation s'appuierait sur d'autres fondations. Je ne revendique pas une exemplarité. Les mathématiques sont la formation que je ne peux effacer.

N.M. : Je voudrais que vous essayiez d'aller plus loin dans cette réponse et jusqu'à la première question que je vous ai posée : comment pensez-vous l'appartenance à la cité, comme vous l'avez dit, c'est-à-dire l'engagement? Est-ce qu'il n'y avait pas aussi, dans le fait «d'inventer un enseignement »-je reprends votre expression - une façon d'avoir une présence pour ainsi dire militante au sein même de l'institution «Jussieu » ? Est-ce que ce n'était pas quand même quelque chose qui était important pour vous, y compris dans votre réflexion picturale? Je voudrais aussi vous interroger sur cette période de votre vie dont vous ne parlez jamais ou presque, la période 68, pendant laquelle vous avez eu une position assez active à la faculté des sciences. J'aimerais que vous essayiez de me dire comment vous pensiez cette sorte de dualité que vous avez entre scientifique et politique, et par rapport à la peinture, bien sûr.

Maurice Matieu : Je ne crois pas qu'il y ait eu cette dualité entre politique et sciences. Parce que, si j'avais eu la possibilité de maintenir ces deux axes à l'intérieur des sciences, je ne les aurais sûrement pas quittées. En fait, il y a eu, pour moi, une impossibilité de maintenir cette cohésion. Je l'ai écrit ailleurs : quand je rentrais au Collège de France, j'avais le sentiment de prendre un autre habit, c'est-à-dire de quitter la rue et de ne pas faire rentrer la rue avec moi là où j'étais censé travailler. Cela m'était insupportable, jusqu'au jour où je n'ai pas pu entrer à l'intérieur du Collège de France.

N.M. : Alors, du coup, que pensez-vous de 68 ? N'empêche qu'à ce moment-là, vous aviez quand même le même habit.

Maurice Mathieu : Je parle en mon nom. Mon engagement est un engagement personnel, ce n'est pas un engagement de parti. À cause de ma date de naissance, j'ai connu la proximité de la guerre 39-45, l'Occupation, la présence allemande. J'ai vu certains événements étant enfant, en ayant le regard au niveau du ceinturon où j'ai lu : «Gott mit und ( ( Dieu est avec nous »). Puis, adulte, adulte conscient, je me suis retrouvé plongé dans une guerre où je ne ressentais aucune possibilité d'adéquation et que du mépris pour le peuple qui la faisait. J'avais en tête cette phrase de Sartre dans Situations : «En 1943, rue Lauriston, des Français criaient d'angoisse et de douleur; la France entière les entendait. L'issue de la guerre n'était pas certaine et nous ne voulions pas penser à l'avenir ; une seule Chose nous paraissait impossible : qu'on pût un jour faire crier des hommes en notre nom. Impossible n'est pas français : en 1958, à Alger, on torture régulièrement, systématiquement, tout le monde le sait, de M. Lacoste 
aux cultivateurs de l'Aveyron, personne n'en parle ${ }^{1}$. » La décision est avant, après il est trop tard.

Évidemment, dans les manifestations, dans l'opposition à la guerre d'Algérie, le peuple s'est retrouvé. Mais je n'ai pas eu la possibilité de rencontrer un appareil politique qui pouvait concrétiser ce sentiment. Il y avait dans les pratiques des appareils politiques de l'époque un opportunisme tactique insupportable.

En 68, j'ai cru que, dans la mobilisation de la jeunesse, il pouvait y avoir, comment dire, une rencontre entre une jeunesse et une pensée qui n'avait pas trouvé son mode d'expression. Cela a été une désillusion puisque la jeunesse, à juste titre, était libertaire et voulait se débarrasser de tous les carcans qui l'opprimaient, dans une exigence légitime d'un vécu immédiat. J'étais déjà à leurs yeux, je ne veux pas dire un vieux con - car la distance d'âge qui nous séparait était modeste -, mais... je n'ai pas pu rencontrer ce lien avec la jeunesse. Donc, 68 n'a pas été pour moi une joie. Si j'en garde une image, personnelle et rayonnante, $c^{\prime}$ est l'absence de voitures dans les rues de Paris.

N.M. : Vous avez quand même été président du comité de grève de Jussieu.

Maurice Matieu : Bon, oui, avec d'autres. Il ne faut pas faire jouer à des individus des rôles particuliers dans un mouvement qui était un mouvement d'ampleur et constamment renouvelé. On a essayé, avec d'autres, d'agir, mais on n'y a pas réussi.

N.M. : Vous avez essayé de faire une rencontre? Une rencontre de type politique?

Maurice Matieu : Cela a été une rencontre impossible et pourtant, évidemment, une volonté politique. J'avais l'espoir de pouvoir affirmer les idées, de voir naître les idées qui n'avaient pas pu s'exprimer dans le combat qui avait été mené au moment de la guerre d'Algérie. J'ai cru que la jeunesse allait être une jeunesse politisée. J'avais de fait une position idéaliste. Les années qui ont précédé l'arrivée de Giscard à la présidence ont été déterminantes. J'ai rejoint les mouvements maoïstes parce que l'important était qu'une discussion collective s'effectue, ne seraitce qu'à partir du Petit Livre rouge, même si - préférant Héraclite - je ne lui accordais que peu de crédit. Ce qui ne m'a pas empêché d'agir. Je ne regrette pas ce que j'ai fait dans ces années post-68, en tentant entre autres d'établir une horizontalité dans les relations, en rencontrant le questionnement de Deleuze avant de l'avoir lu : vouloir casser toute verticalité hiérarchique en créant des réseaux horizontaux. Ces réseaux horizontaux ont été une réussite dans quelques lieux, bien sûr, géographiquement limités.

N.M. : Cela veut dire qu'il y a chez vous plusieurs axes d'actions. Le domaine scientifique était strictement limité...

1 Sartre, J.-P., 1959. Situations, III, Paris, Gallimard.
Maurice Matieu : Très limité à l'institution... et à la réussite dans l'institution...

N.M. : Mais aussi à une certaine éthique qui vous conduisait toujours à introduire le peuple plutôt que l'élite ou plutôt que le pouvoir. Ce que vous venez de dire de l'enseignement, de la guerre d'Algérie et même des années 68 semble montrer que la part du scientifique comptait beaucoup moins que celle qui était proprement politique, une espèce de défi politique. Et la peinture là-dedans, qu'est-ce qu'elle devenait, la peinture?

Maurice Matieu : La peinture, très vite, quand je me suis noyé à l'intérieur, j'ai compris que c'était un terrain éminemment politique. Il y a peu de grands tableaux - il y a beaucoup de tableaux -, mais il n'y a pas de tableau fondamental qui ne soit pas politique. Prenons l'exemple des grands éclats : le Christ en croix pourri du Retable d'Issenheim, Vélasquez et Les Ménines, La Crucifixion du Tintoret à Venise, La Ronde de nuit de Rembrandt dans les pays du Nord... À chaque fois qu'un tableau témoigne, traverse le temps, la dimension politique qui l'emporte est déterminante. Et comme la peinture a toujours été une expression immédiate et surveillée, pour arriver à son expression, ou plutôt pour arriver à exprimer sa volonté, il a toujours été nécessaire que la peinture utilise le subterfuge. Ces subterfuges sont les axes fondamentaux de la vitalité politique.

N.M. : Et pour vous, dans votre peinture?

Maurice Matieu : Pour moi, je n'ai rien à dire. Je peux affirmer une volonté. Je ne peux pas prétendre, moi-même, que ma peinture ait un sens, et surtout un sens politique.

N.M. : Vos titres, en tout cas, ont toujours été des titres politiques depuis L'Impossibilité de peindre la guerre jusqu'à La Banalité du massacre...

Maurice Matieu : Certes, ce sont des titres politiques, mais qui ne témoignent pas d'une réussite, d'une ouverture politique. J'ai choisi cet axe, ce qui ne veut pas dire que j'ai réussi. En tout cas, je n'y vois que ma volonté, je n'y vois pas le regard des autres.

N.M. : Puis-je me permettre de dire que, dans la réalisation, la volonté politique est prioritaire dans votre esprit, que la peinture est, pour vous, le lieu privilégié de l'effort de création politique? Puis-je me permettre de dire cela?

Maurice matieu : C'est le seul lieu, pour l'instant, où j'ai le sentiment d'avoir une adéquation entre ma vie et mon travail. Pour l'instant, c'est le seul lieu. Peut-être y en aura-t-il d'autres, peut-être vais-je découvrir l'écriture? J'ai encore longtemps à pouvoir espérer expérimenter. Peut-être retournerai-je - ce qui m'étonnerait parce que les gammes sont trop longues à refaire - vers les mathématiques...

N.M. : Les mathématiques, vous y avez renoncé finalement, du moins dans la relation au politique?

Maurice Matieu : Non, non, je n'y ai pas renoncé, je me sers des mathématiques d'une manière continue. 
D'abord parce que dans les moments d'essoufflement, pour reprendre mon souffle, je reviens sur les travaux antérieurs sur lesquels j'ai réfléchi à la recherche des traces d'une idéologie cachée toujours rémanente. Sans en avoir conscience, on est des agents de propagande. J'essaie évidemment de traquer ces traces chez moi, et c'est là que les mathématiques me servent. C'est un double moyen de réflexion, mais il y a toujours un versant appauvri. Prenons l'exemple de Pevsner, un sculpteur : ses sculptures sont des surfaces réglées, c'est-à-dire des surfaces engendrées par des droites. Or, ce qui est porteur de sens, ce n'est pas l'objet construit par Pevsner, mais l'équation mathématique de la surface. Pour moi, les mathématiques sont une méthode. Il faut que je sois sûr que le point ultime de signification soit bien là où je veux qu'il soit.

N.M. : Les mathématiques, comme une sorte de contrôle de votre propre «insoumission »?

Maurice Matieu : C'est un moyen de vérification, ce qui ne veut pas dire que je n'utilise pas, stricto sensu, les mathématiques dans un tableau. En voici un exemple : dans son film Shining, Kubrick dispose ses multiples labyrinthes à l'intérieur et à l'extérieur de l'hôtel de Denver, construit sur un sanctuaire d'Indiens, pour bien montrer qu'il installe son film précisément là où la littérature, selon lui, a renoncé à le faire, c'est-à-dire sur le mythe. L'ouverture du film, véritable ouverture d'opéra, dans les dédales du Colorado en témoigne. Au fond d'un couloir de l'hôtel, loin, les portes d'un ascenseur s'ouvrent et déversent un flot rouge - image que j'associe évidemment à du sang. Ce qui me pose la question de savoir combien d'hommes nous ont précédés. Voilà comment opère un détail : il faut que je reconstitue le chiffrement et les mathématiques se réinstallent. J'inverse la démographie pour trouver environ 75 milliards d'individus, soit 375 milliards de litres de sang. Mais comment rendre sur la toile le sentiment d'un effacement par cette profusion? J'ai utilisé la méthode de construction que donne Hilbert de la courbe de Péano, courbe qui passe par tous les points d'un carré sans se couper, en faisant des partitions du carré par multiples de 2 , de 3, de 5 et de 7 . Quand on augmente le nombre de partitions des côtés du carré, la courbe devient indiscernable et fait tache (Dessin).

N.M. : Il y a aussi, dans ce recours aux mathématiques, l'idée d'aller à l'inverse d'un ordre.

Maurice Matieu : J'ai tenté de repérer toutes les contraintes idéologiques que je subis quotidiennement, depuis le miroir jusqu'à l'alignement des lames de parquet. Il m'a fallu trouver des pavages, des pavages du plan, qui ne définissent pas de direction privilégiée. J'en ai trouvé un certain nombre que j'ai construit à la règle et au compas sans avoir pensé à utiliser des mathématiques plus élaborées. C'est en voyant dans un champ un grillage d'ursus déformé par l'entêtement des moutons à vouloir s'échapper, en regardant ce grillage, que j'y ai trouvé

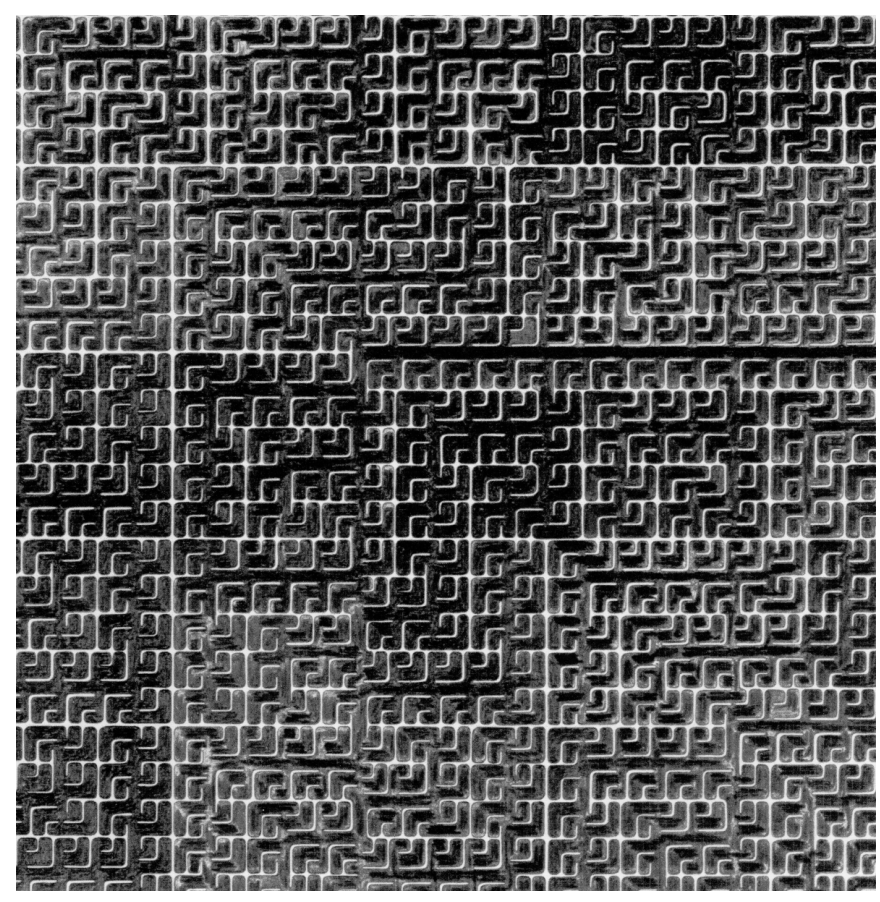

Dessin. Peano crayon sur arches $(93 \times 62)$, Maurice Matieu, 1985-1986.

la possibilité de construire un pentagone qui recouvrait la totalité du plan sans définir de direction privilégiée. Quelques années plus tard, j'ai appris avec plaisir que le mathématicien Penrose en faisait la théorie. Tout cela pour $1 \mathrm{~m}^{2}$ de la toile que Jean Borreil nommera La Mirada ou la Tendresse du regard.

N.M. : Je sais que tout est très fortement apprêté dans votre pensée et dans votre réalisation. Mais, dans une certaine mesure, ce que je cherche à comprendre, c'est ce qui est premier, le moteur dans votre pensée et dans votre travail. Et en reprenant le terme d'engagement - même s'il n'est plus très juste -, je me demande si, compte tenu de ce que vous venez de dire, ce qui est premier n'est pas toujours le politique. Et ensuite, qu'est-ce qui viendrait en second, puis en troisième? Dans cette trilogie - que j'essaie de clarifier pour vous-même et pour ceux qui aiment votre travail : le politique, les mathématiques et la peinture, dans cette sorte de triangle avec ses trois pôles -, par quoi vous commencez-vous? Comment les enchaînez-vous? Quelle est la relation qui s'établit entre ces trois pôles?

Maurice Matieu : Roger Martin du Gard rapporte, dans son petit livre Notes sur André Gide, comment selon lui il écrit :

"Pour mieux se faire comprendre il [André Gide] a pris une feuille blanche, y a tracé une ligne horizontale toute droite. Puis, saisissant ma lampe de poche, il a promené lentement le point lumineux d'un bout à l'autre de la ligne : - "Voilà votre Barrois, voilà vos Thibault [...]. Vous imaginez la biographie d'un personnage, ou l'historique d'une famille, et vous projetez là-dessus votre lumière, honnêtement, année par année [...]. Moi, voilà comment 
je veux composer mes Faux monnayeurs [...]." Il retourne la feuille, $y$ dessine un grand demi-cercle, pose la lampe au milieu, et, la faisant virer sur place, il promène le rayon tout au long de la courbe en maintenant la lampe au point central : - "Comprenez vous, cher? Ce sont deux esthétiques. Vous, vous exposez les faits en historiographe, dans leur succession chronologique. C'est comme un panorama qui se déroule devant le lecteur. Vous ne racontez jamais un événement passé à travers un événement présent, ou à travers un personnage qui n'y est pas acteur. Chez vous, rien n'est jamais présenté de biais, de façon imprévue, anachronique. Tout baigne dans la même clarté, direct, sans surprise. Vous vous privez de ressources précieuses! [...]. Pensez à Rembrandt, à ses touches de lumière, puis à la profondeur secrète de ses ombres. Il y a une science subtile des éclairages; les varier à l'infini, c'est tout un art $^{2} . "$ "

Quant à moi, sur la feuille de papier, je trace un triangle dont les sommets sont la peinture, la mathématique et le politique. Mais je ne tiens pas la lampe, on la tient pour moi et je vois, suivant les périodes de ma vie, un sommet du triangle éclairé. Le sommet éclairé devient essentiel et oriente mon travail.

N.M. : Ce qui explique votre intérêt pour l'Irak et vos dernières toiles où les zelliges tracent L'Infini sous les pieds?

Maurice Matieu : L'Irak est un bon exemple de ce rapport indémêlable entre la science, l'art et le politique. Je ne veux pas revenir sur la situation actuelle et la honte de cette guerre. Il y a une banalité répétée sur tous les tons, le judéo-christianisme qui n'explique rien. Quand je me suis trouvé la première fois en Irak, j'ai retrouvé le manuel, Malet et Isaac, de la classe de sixième où l'enseignement de l'histoire débutait par les influences gréco-assyro-babyloniennes. Nous n'avons pas su nous détacher des mesures d'espace et de temps introduites par les Sumériens. C'est devenu une évidence, peut-être une nostalgie, mais je ne le crois pas : effectivement, la part gréco-mésopotamienne est prépondérante, mais notre culture est romaine. Nous nous sommes construits dans l'espace géographique romain. C'est une illusion de croire que le judéo-christianisme a défini cet espace géographique. Ce n'est pas vrai. La culture islamique a été dominante, la présence de savants juifs, importante. N'empêche que l'essor a été occidental et que l'islam n'a pas suivi. L'Occident a hérité de la culture romaine, c'est-à-dire la présence de la technique dans la culture.

Je suis allé en Irak par engagement. Je considérais que l'attitude à l'égard du peuple irakien, pendant l'embargo, était insupportable. Je ne comprenais pas pourquoi, au Chili, on avait su dissocier Pinochet du peuple chilien, qu'il était légitime de soutenir, et que, par contre, aucune différence n'était faite entre le peuple irakien et Saddam Hussein. Pourquoi, dire qu'on soutenait le peuple irakien était considéré comme potentialité fasciste? On ne peut pas continuer à supporter l'humiliation des peuples arabes. Avoir comme image de cette culture celle des gouvernants corrompus et, comme image des peuples, celle des immigrés. L'urgence est de rétablir un regard sur cette culture.

Je me suis donc intéressé aux zelliges parce que j'y ai $\mathrm{vu}$, non pas seulement l'usage qu'en ont fait les derviches, c'est-à-dire un désespoir de l'infini, témoin d'une immobilité sur laquelle on ne peut que danser et tourner, mais la possibilité de déstructurer notre conception de l'espace et d'y importer un nouvel infini qui n'est pas repoussé mais sous les pieds.

N.M. : En même temps, c'était une sorte de réhabilitation?

Maurice Matieu : Non, ce n'est pas une réhabilitation, c'est seulement dire que cette culture est une grande culture. Quand Ibn Khaldoun dit qu'il est témoin de trois religions, toutes trois liées à l'écriture : l'islam, le christianisme, le judaïsme, auxquelles il ajoute la culture de la Grèce, il est étonné de voir des civilisations aussi extraordinaires que la civilisation mésopotamienne ou la civilisation égyptienne ne pas avoir de présence. Il ajoute que, même si on pouvait reconstituer la totalité de leurs textes, notre inertie est telle qu'on ne saurait quitter ces quatre pensées écrites.

N.M. : Je lancerai, pour mettre fin à cet entretien, une dernière provocation : dans le fond, il y a quelque chose de syncrétique dans votre façon de penser et de peindre.

Maurice Matieu : L'homme est le point central, c'està-dire l'énormité du travail qu'il a effectué. Nous sommes face à cette énormité. Il faut voir, savoir, pour faire.

${ }^{2}$ Martin du Gard, R., 1951. Notes sur André Gide, Paris, Gallimard. 